nature

immunology

\title{
Lighting the way forward
}

\section{Although recent progress has aided our understanding of the processes that prevent immune tolerance breakdown, this Focus issue illustrates how much remains unknown about susceptibility to and pathogenesis of autoimmune diseases.}

J ervading the seminars and discussion sessions at recent immune tolerance and autoimmunity conferences is the realization that any given autoimmune disease may in fact represent a collection of disorders of different etiologies that result in somewhat similar clinically recognizable pathologies. The heterogeneity of any one autoimmune disease is reflected in the difficulty of diagnosing and treating many diseases. Diagnosing systemic lupus erythematosus, for example, requires that a patient show 4 or 5 out of 11 clinical characteristics associated with the disease. With regard to treatment, as many as $20 \%-30 \%$ of rheumatoid arthritis patients fail to improve after treatment with tumor necrosis factor (TNF)-neutralizing antibodies. Although variation in the time of presentation and therapy during the course of disease progression may certainly contribute to this heterogeneity in diagnosis and responsiveness to treatment, variation in actual etiology is likely to play a major role. Genome-wide association studies certainly suggest a heterogeneous and combinatorial contribution of genetic factors to autoimmune disease susceptibility. For example, more than 16 loci have been linked to rheumatoid arthritis susceptibility so far. Thus, in autoimmune disease, there seem to be few absolutes.

For this reason, the articles in this Focus issue (http://www.nature. $\mathrm{com} / \mathrm{ni} /$ focus/tolerancebreakdown/) cover what is known about individual mechanisms responsible for preventing immune tolerance breakdown, rather than what is known about individual autoimmune diseases. However, as highlighted by Diane Mathis and Christophe Benoist in the Focus Overview article, this summary of our current understanding of the cellular and molecular players mediating immune tolerance may prove in retrospect to be preliminary at best. Fortunately, many of the Reviews in this Focus issue prominently acknowledge these gaps in knowledge and suggest avenues of investigation for further consideration.

Shimon Sakaguchi and Kajsa Wing assess our current understanding of how regulatory $\mathrm{T}\left(\mathrm{T}_{\text {reg }}\right)$ cells promote immune tolerance by suppressing the activation of self-reactive conventional $\mathrm{T}$ cells, and the role of the transcription factor Foxp 3 in $\mathrm{T}_{\text {reg }}$ cell function. They highlight the fact that we still do not fully understand what signals are needed to turn on Foxp3 expression or to initiate $\mathrm{T}_{\text {reg }}$ cell lineage commitment in immature thymocytes. Picking up the theme of lymphocyte development, Harald von Boehmer and Fritz Melchers discuss the series of developmental checkpoints responsible for purging the B and $\mathrm{T}$ lymphocyte repertoire of autoreactive clones, and/or for diverting these autoreactive clones into unconventional lymphocyte lineages. As many autoreactive T lymphocytes slip through these checkpoints by evading deletion and/or lineage diversion, William Mueller reviews our current understanding of peripheral mechanisms responsible for controlling the activity of such autoreactive T cell escapees. Lastly, Alexander Chervonsky covers the influence, only recently appreciated and perhaps surprising, of commensal and pathogenic microbes on immune tolerance and autoimmunity. Although many commensal microbes are not culturable in established conditions and thus remain largely mysterious, recent data linking one such organismsegmented filamentous bacterium-with proinflammatory gene expression in the mouse intestine will likely spark efforts to more intensively examine and understand these organisms.

Also included in this Focus issue are two opinionated Perspective articles. Lawrence Steinman rightly expresses concern over and discusses the fact that studies of animal models of human autoimmune disease and studies of the human diseases themselves often reach discrepant conclusions. Casey Weaver highlights the concept that dividing immune cell populations into neat, clearly demarcated subsets-a practice that understandably comes as second nature to many immunologists - might not accurately reflect or aid our understanding of the flexible and dynamic immune cell populations responsible for driving and inflicting tissue damage in patients with autoimmune disorders.

Running throughout all articles in this Focus issue are some common themes that illuminate areas in need of further investigation. We must try to decipher the processes influencing initial tolerance breakdown and to distinguish them from processes responsible for perpetuating autoimmune pathology. Some elements of diseases classified as autoimmune might not involve any actual self-reactivity. Whether commensal organisms should be considered as 'self' has not yet been determined. The influence of target organ physiology on many organ-specific autoimmune diseases is not yet fully understood. Formation of multidisciplinary research teams incorporating immunologists as well as experts on joints, the heart, the skin, the kidney and the pancreas may help propel forward our comprehension of the factors influencing pathology of specific autoimmune disorders.

There is no doubt that much of the value generated by this Focus issue is derived from its candid assessment of what we don't know. However, we should bear in mind that our knowledge of the immune system is expanding at an increasingly rapid pace and that immunologists are adjusting their views accordingly. Peppered throughout the pieces in this issue are statements reminding us of past dogmas that in hindsight seem simplistic and perhaps even naive (for example, the $\mathrm{CD} 4^{+} \mathrm{T}$ cell universe was once thought by many to consist solely of $\mathrm{T}_{\mathrm{H}} 1$ and $\mathrm{T}_{\mathrm{H}} 2$ cells). In that respect, the real measure of our future progress in unraveling the mechanisms facilitating immune tolerance and avoidance of autoimmunity may in fact be whether or not the next Focus on immune tolerance includes statements correcting and revising some viewpoints put forward in this one. 